\title{
Development of Three-dimensional Measurement System for Earth Retaining Wall for Excavating Work
}

\author{
Takaki MATSUMARU \\ Kenichi KOJIMA \\ Foundation \& Geotechnical Engineering Laboratory, Structures Technology Division \\ Yuji TANAKA \\ Ryosuke KURIYAMA \\ JR East Consultants Company, Fukken Engineering Co., Ltd., \\ (Formerly Foundation \& Geotechnical Engineering Laboratory) \\ Toshiyasu HISASHIMA \\ East Japan Railway Company \\ Yuki KOMINATO \\ West Japan Railway Company
}

\begin{abstract}
Monitoring of the deformation of earth retaining walls for excavating work is vital in order to protect the surrounding environment and to ensure the safety of structures during construction. Since it is difficult to evaluate the overall behavior with only partial measurement, it is expedient to introduce multipoint measurement. However, this method tends to be expensive. This paper introduces a system developed for evaluating and visualizing retaining walls as a three-dimensional curved surface. In order to confirm the effectiveness of the proposed system in actual monitoring, the system was applied to the on-site measurement. This paper also proposes a method of monitoring retaining walls by combining this system and simple inclinometers.
\end{abstract}

Keywords: earth retaining wall for excavating work, cubic B-spline function, inclination, stand-alone inclinometer

\section{Introduction}

Monitoring of the deformation of earth retaining walls for excavation is vital in order to keep the surrounding environment and structures safe during construction. Furthermore, in construction sites close to rail tracks, the limit value for the displacement of the retaining wall is very small. However, there are certain problems in monitoring of earth retaining walls. For example, it is difficult to evaluate the overall behavior of the retaining walls by means of partial measurement using plum bobs, and multipoint measurement using multi-element inclinometers tends to be expensive.

With these problems in mind, a system was developed for use in evaluating and visualizing retaining walls as a three-dimensional curved surface. In this system, the cubic B-spline function is adopted as the analytical technique, which is used to describe the shape of land as a threedimensional curved surface based on sets of data for the elevation altitude as proposed by Nonogaki et al. [1]. A method was proposed for evaluating inclinometer data as a surface without transforming incline into displacement. The validity and the adequacy were confirmed by applying the proposed system to field measurement. A method of monitoring walls by combining this system and standalone inclinometers was also proposed.

\section{Three-dimensional measurement system for re- taining walls}

In the developed three-dimensional measurement system for retaining walls, the information obtained from measurement equipment is recorded in real-time, the discrete data was analyzed so that it can be continuous data, and the deformation of the retaining wall is visualized as a three-dimensional surface. Figure 1 shows the developed system.

This system consists of three parts: recording data (1), analyzing data (2), and visualizing the deformation of retaining walls as three dimensional surfaces (3). In recording data (1), the data obtained from measurement equipment is passed to analytical part. The usable measured data in this system refers to displacement and inclination. The usable types are the time-series data recorded by measurement equipment and the irregular data obtained manually such as survey by total stations and photo-measurement.

In analyzing data (2), the deformation of the retaining wall is analyzed as a three-dimensional surface based on the measured data. The cubic B-spline function is adopted as the analytical technique, which is used to describe the shape of land as a three-dimensional curved surface based on sets of data for the elevation altitude. In order to make this method applicable to the evaluation of deformation of retaining walls, the method was improved so that incline can be used for evaluating the deformation, and the accuracy of each piece of measurement equipment can be considered.

In visualizing the deformation as a three-dimensional surface (3), the analyzed data relating to the surface in (2) is described with a three-dimensional viewer. For the description technique, the graphic tool included in OS is used.

In this system, the optimal arrangement for measurement equipment can be studied before starting excavating 


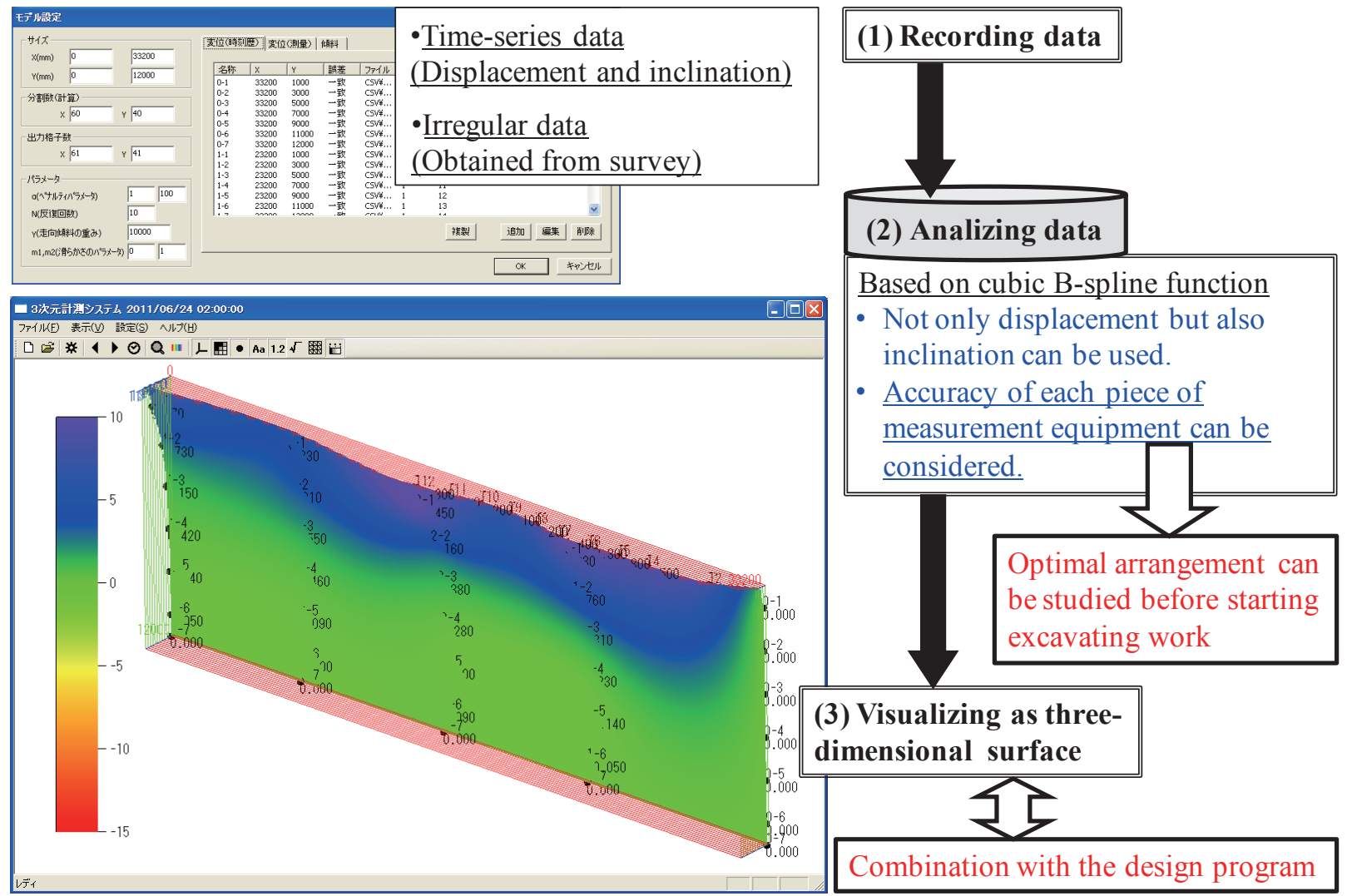

Fig. 1 Three-dimensional measurement system

work [2]. Furthermore, in combination with the design program for retaining walls, the observational construction which combines design and measurement can be easily conducted [3].

\section{Outline of the analytical method}

In order to analyze and visualize the deformation of retaining walls, evaluating data obtained from measurement equipment as a three-dimensional curved surface is necessary. In this paper, the cubic B-spline function developed by Nonogaki et al. [1] was adopted for the measurement of the retaining wall.

\subsection{Cubic B-spline function}

Figure 2 shows the three-dimensional coordinate space for describing the deformation of the earth retaining wall. In this figure, the $x, y$, and $z$ axis indicate the direction of the retaining wall, the depth, and the direction in which the wall deforms respectively. The earth retaining wall is expressed as a smooth and continuous surface defined by

$$
f(x, y)=z
$$

In the cubic B-spline function, the region for drawing the surface is divided into the equally-spaced areas $M_{\mathrm{x}}$ and $M_{\mathrm{y}}$ in $x$ and $y$ axis. By setting the $M_{\mathrm{x}}+7$ and $M_{\mathrm{y}}+7$ equallyspaced nodes, the surface is expressed by

$$
f(x, y)=\sum_{i=1}^{M x+3} \sum_{j=1}^{M v+3} c_{i j} N_{i}(x) N_{j}(y)
$$

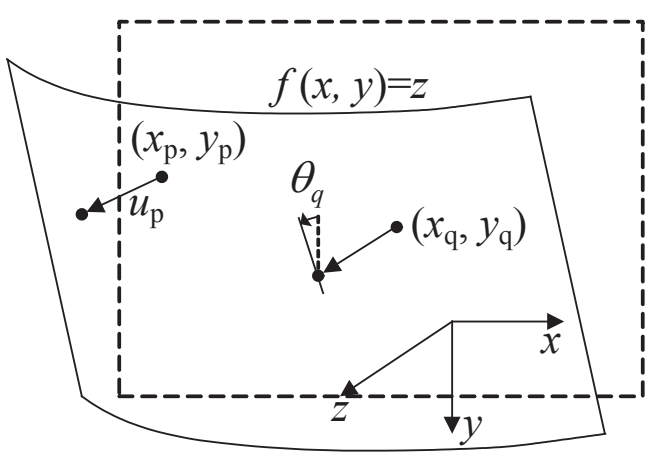

Fig. 2 Three-dimensional coordinate space for drawing deformation of the retaining wall

where $N_{i}(x)$ and $N_{j}(y)$ are the cubic B-spline function, and $c_{\mathrm{ij}}$ is an unknown coefficient.

In order to determine the surface, (2) is solved as the optimization problem from a lot of surfaces which satisfy the displacement data at each measurement point, based on the exterior penalty function method. Specifically, the extended objective function $Q$ is defined by

$$
Q(f ; \alpha)=J(f)+\alpha R(f)
$$

where $J(f)$ is the function for evaluating the smoothness of the surface, $R(f)$ is the function which expresses the sufficiency degree of data, and $\alpha$ is the parameter for balancing these two functions. The optimal surface is determined by substituting $c_{\mathrm{ij}}$ into (2) obtained from $\partial Q(f ; \alpha) / \partial c_{\mathrm{ij}}=0 . J(f)$ is written by the function proposed by Shiono et al [4]. 
The function that expresses the sufficiency, $R(f)$, is mentioned below. The coordinates $\left(x_{\mathrm{p}}, y_{\mathrm{p}}, z_{\mathrm{p}}\right)$ where measurement equipment is placed, and the measured displacement $u_{\mathrm{p}}$ have the following relationship.

$$
f\left(x_{p}, y_{p}\right)=z_{p}+u_{p}
$$

Therefore, using the square average of the error $\varepsilon_{\mathrm{p}}$ between the curved surface and the obtained displacement data, $R(f)$ is evaluated as

$$
\begin{gathered}
R(f)=\sum \varepsilon_{p}^{2} / n_{h} \\
\varepsilon_{p}=\sum_{i=1}^{M x+3} \sum_{j=1}^{M_{y}+3} c_{i j} N_{i}\left(x_{p}\right) N_{j}\left(y_{p}\right)-\left(z_{p}+u_{p}\right)
\end{gathered}
$$

where $n_{\mathrm{h}}$ is the number of data satisfies (4).

\subsection{Use of measured inclination}

When monitoring a retaining wall, inclination is often measured rather than displacement due to the relative ease of doing this. For this reason, it is important to develop a method under which incline data can be used for evaluating deformation.

The function $R(f)$, defined by (5), is divided into two functions, $R_{\mathrm{h}}(f)$ and $R_{\mathrm{d}}(f) . R_{\mathrm{h}}(f)$ expresses the sufficiency degree of displacements, and $R_{\mathrm{d}}(f)$ expresses that of inclines. Using these functions, $R(f)$ is expressed as

$$
R(f)=R_{h}(f)+\gamma R_{d}(f)
$$

where $\gamma$ describes the weight of the sufficiency degree of incline. $R_{\mathrm{h}}(f)$ is expressed by (5).

$R_{\mathrm{d}}(f)$ is defined as follows. At the position where an inclinometer is located, $\left(x_{\mathrm{q}}, y_{\mathrm{q}}, z_{\mathrm{q}}\right)$, the derivative of the function $f$ with respect to $y$ is described by the following equation.

$$
f_{y}\left(x_{q}, y_{q}\right)=-\tan \theta_{q}
$$

Therefore, the function $R_{\mathrm{d}}(f)$ is expressed as

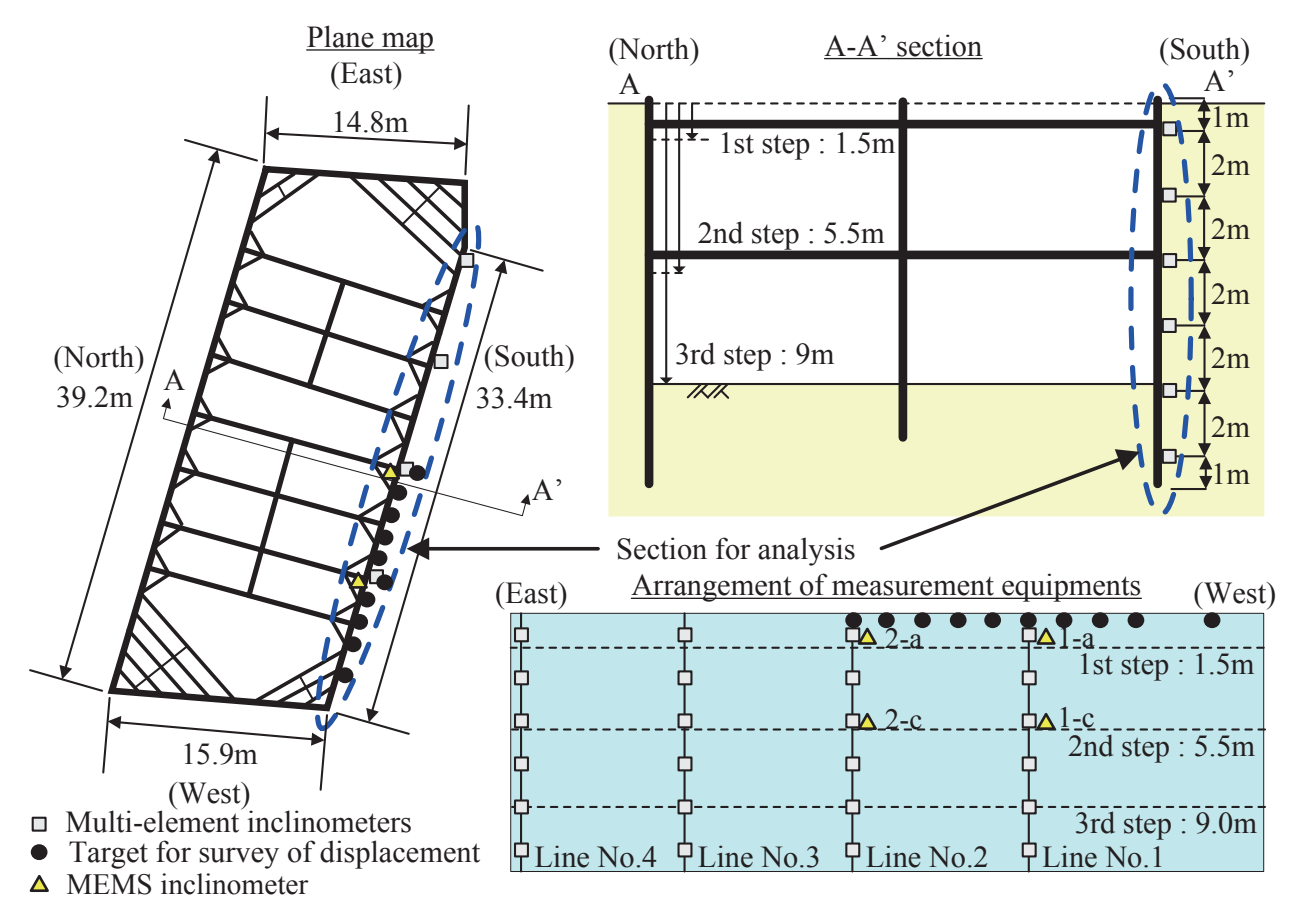

Fig. 3 Conditions of field construction and measurement

$$
R_{d}(f)=\sum\left\{\sum_{i=1}^{n_{d}} \sum_{j=1}^{M x+3} c_{i j} N_{i}(x) N_{j}^{\prime}(y)+\tan \theta_{q}\right\}^{2} / n_{d}
$$

where $n_{\mathrm{d}}$ is the number of the obtained incline data.

\section{Adaptation of the proposed method for field mea- surement}

\subsection{Field condition of construction and measure- ment}

Figure 3 shows the field conditions of construction and arrangement of measurement equipment. The excavating work was conducted at a site $39 \mathrm{~m}$ by $16 \mathrm{~m}$ to a depth of 9 $\mathrm{m}$. The surface layer of the ground was a very soft alluvial clay layer about $13 \mathrm{~m}$ in thickness, with a small $\mathrm{N}$-value of the standard penetration test, followed by a gravel layer with an $\mathrm{N}$-value of 50 . The retaining walls featured a bracing method with intermediate piles. The wall material was steel sheet piles. The excavating work consisted of three steps as shown in Fig. 3.

Wall monitoring was conducted in the south section in order to keep the existing tunnel safe for traffic. Monitoring was conducted using multi-element inclinometers. As shown in Fig. 3, there were four survey lines and six inclinometers were set on each line. To check the monitoring data, a survey of the displacement of the wall using the total station (TS) was also conducted around line no. 1 and line no. 2 when each step of excavating work was finished.

\subsection{Evaluation of the deformation of retaining walls}

The deformation of the wall in three-dimensional space was evaluated and visualized in the region approx. 33.4 $\mathrm{m}$ wide, covered by dotted lines in Fig. 3. Two series of arrangements of the measurement equipment were consid- 


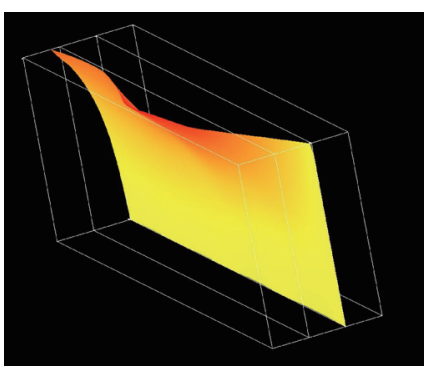

(a) After the 1st step

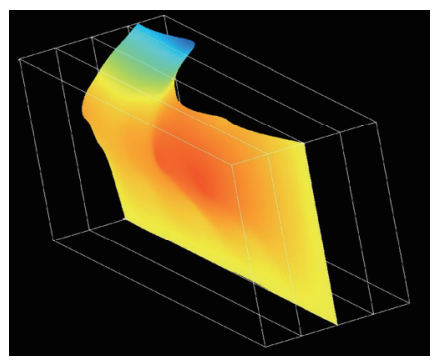

(b) After the 2nd step

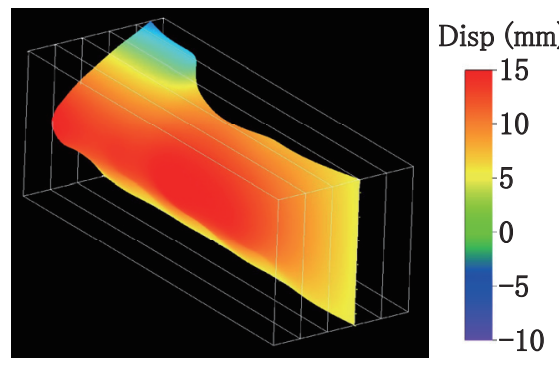

(c)After the 3rd step

Fig. 4 Evaluated and visualized three-dimensional deformed surface of a retaining wall

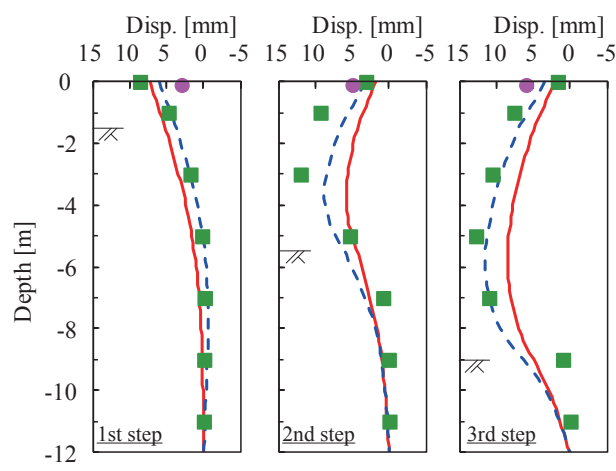

(a) Line no. 1

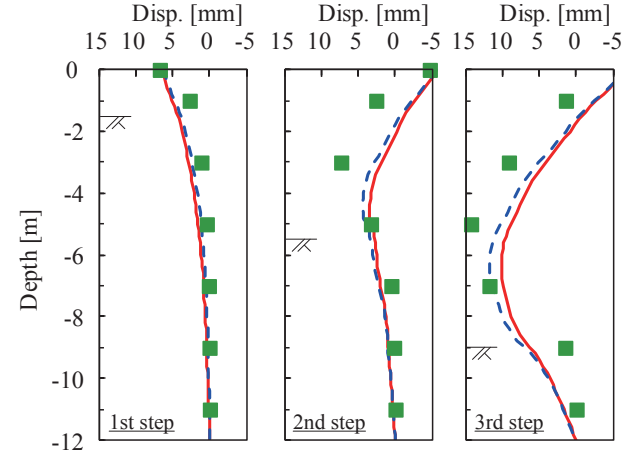

(b) Line no. 4

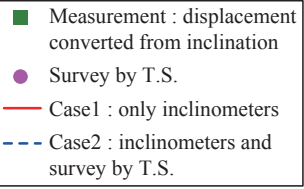
survey by T.S.

Fig. 5 Distribution of the displacement at each excavation step

ered. In case 1 , only 24 multi-element inclinometers were used. In case 2 , not only the data obtained by inclinometers but also the displacement data obtained from the survey with TS was used.

Figure 4 shows the deformed wall which was evaluated and visualized after the excavation at each step in case 1 . From the beginning to the end of the work, the deformation of the wall was represented satisfactorily as a threedimensional surface. While the deformation of the wall increased as the excavating work progressed, the area where the maximum displacement occurred became deeper and the deformation close to the ground level decreased due to the influence of the reaction force generated by installing braces.

Figure 5 shows the distributions of deformation at the cross section of line no. 1 and no. 4 as shown in Fig. 3, after conducting excavation at each step. This figure also shows the displacement converted from the angle obtained from inclinometers and the distances between two inclinometers, and the displacements obtained from the survey by TS. For both cases, the distributions of the displacement evaluated using the proposed method coincide with the mode of directly measured deformation. The distributions of the displacement were changed largely according to results obtained from the survey by TS, as shown in the figure at the cross section no.1, where a lot of TS survey objects were located. For this section, the simulated results of case 2 were close to the surveyed results around the top of the wall.
5. Development of the measurement method using a three-dimensional measurement system and stand-alone inclinometers

The good adaptability with regard to the field measurement confirmed validity of the proposed method. The benefit of this proposed method lies in the fact that it is possible to use the measured inclination without converting it to displacement for analysis and visualization. In the field measurement, in order to produce the measurement lines for evaluating the displacement, multi-inclinometers are often used. This proposed method can therefore produce a high degree of freedom for the arrangement of measurement equipment. Furthermore, a large number of stand-alone inclinometers, such as an inclinometer using a MEMS sensor, have been developed recently [5]. Therefore, by combining the proposed system and the stand-alone inclinometers, it would be possible to achieve precise and inexpensive measurement. This section explains the problems for the adaptation and the trial of the field measurement.

\subsection{Adaptation of stand-alone inclinometer to mea- surement of retaining walls}

In the field mentioned in the previous section, the inclinometers using a MEMS sensor were installed at the same position as the multi-inclinometers, and the accuracy of the inclinometers was checked.

The locations of the inclinometers using MEMS sensors are shown in Fig. 3. The inclinometers of 1-a and 2-a at the top of the ground were installed before staring the excavation, and 1-c and 2-c at a depth of G.L.-5.5 $\mathrm{m}$ were 


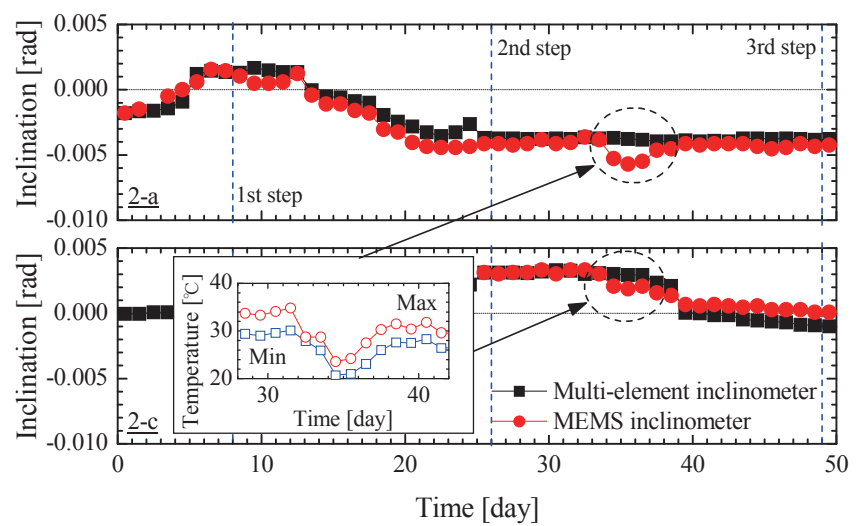

Fig. 6 Time history of the measured inclination

installed after the end of the excavation in the second step. The inclinometers were fixed by welding them to sheet piles on the side where excavating work was conducted.

Figure 6 shows the time histories of the inclination recorded by the inclinometers of 2-a and 2-c. In this figure, the inclinations recorded by multi-inclinometers at the close spots were also plotted. From this figure, it can be understood that the inclinations obtained from each of the two types of inclinometers almost coincided. On the other hand, as shown in the behaviors from the 35th to the 38th, the inclinations obtained from the multi-inclinometers had a variation of about $0.002 \mathrm{rad}$, though the inclinations from multi-inclinometers were not changed. In this figure, the time histories of the minimum and maximum temperatures were also shown. The temperature exhibited low values compared to the temperature achieved before and after this period. The inclination obtained from the MEMS inclinometers demonstrated variation affected by the heat of the sheet piles because the inclinometers were fixed directly to the sheet piles. However, as the general behaviors almost coincided between the two types of inclinometers, the inclinometers using MEMS sensors are applicable to the measurement of the deformation of the retaining wall.

\subsection{Study of arrangement of inclinometers}

When stand-alone inclinometers are used for field measurement, boring is not required but inclinometers cannot be set before the start of excavating work. Therefore, inclinometers would be set as the progress of excavating work.
In this study, the accuracy of the surface simulated was only checked on the basis of the incline data obtained from the inclinometers located above the bottom at each step of excavating work, by using the incline data obtained from the multi-inclinometers as if it were obtained from standalone inclinometers.

For the simulation, only the inclinometers located above the bottom were used, as shown in Fig. 3. The number of the inclinometers was 4 at the first step, 12 at the second step and 20 at the third step. The displacement data obtained from the survey using TS was also used for all steps.

Figure 7 shows the simulated distributions of the displacement. The displacement at line no. 1 was quite similar to that obtained by using all inclinometers, which corresponds to the result of case 2 in the previous section. In contrast, the displacement at line no.4 was quite different. These results indicated that it is difficult to reproduce the deformation of the wall using only a limited number of inclinometers.

For this reason, in the figure of line no. 4, the result simulated using all multi-inclinometers located at line no. 3 was also shown. In this simulation, the measurement was considered to be conducted using multi-inclinometers at one survey line. This result almost coincided with the results obtained using all multi-inclinometers.

From these simulations, the measurement using the proposed system and stand-alone inclinometers could be combined provided that direct measurement of displacement or installation of one survey line for multi-inclinometers is installed.

\subsection{Adaptation for field measurement}

Under consideration of the studies mentioned in the previous section, the validity of conducting the measurement using the proposed system and the stand-alone inclinometers was examined. Furthermore, the cost of the measurement was also estimated.

\subsubsection{Condition of the constructing field and mea- surement of retaining walls}

Figure 8 shows the condition of the constructing field and measurement of retaining walls. The object of the measurement was the retaining wall for constructing a pit for

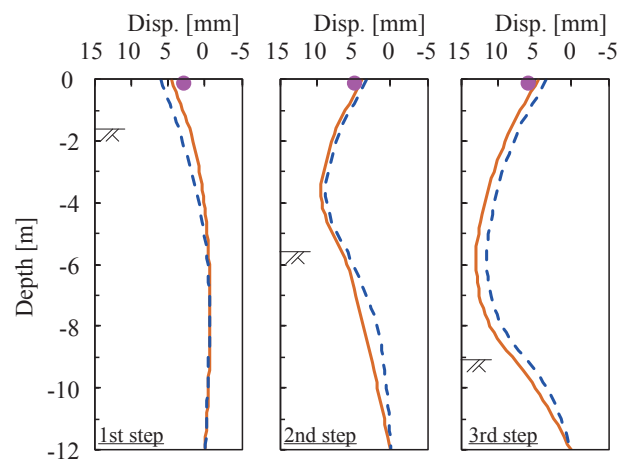

(a) Line no. 1

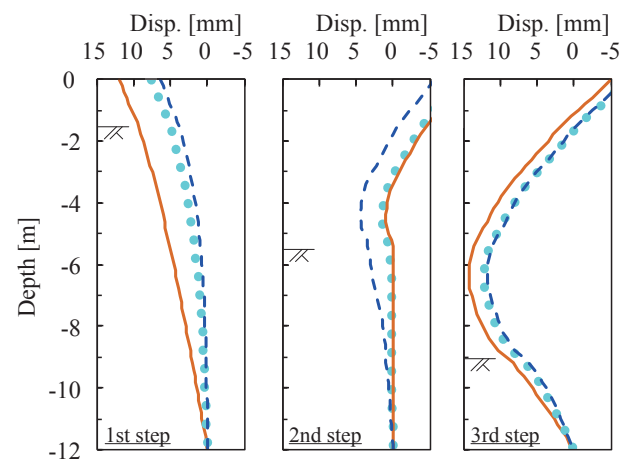

(b) Line no. 4

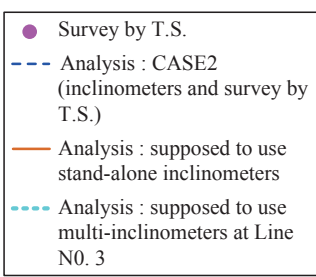

N0. 3

Fig. 7 Distributions of the displacement at each excavation step 


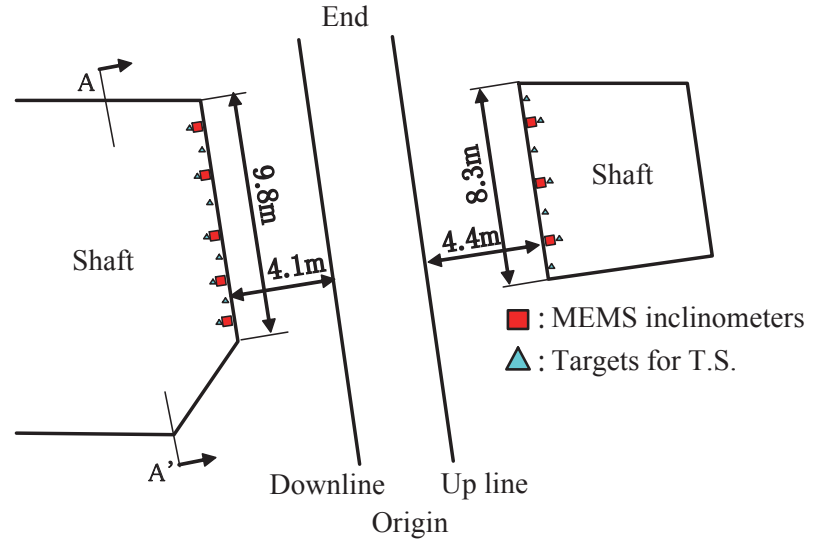

Fig. 8 Conditions of field construction and measurement

a culvert structure under a rail track. The material of the retaining walls was soil-cement and the cantilever method was adopted. The depth of the excavation was $3.0 \mathrm{~m}$ and the distance between the retaining wall and the rail track was between $4.1 \mathrm{~m}$ and $4.4 \mathrm{~m}$.

The surface layer of the ground was a very soft alluvial clay layer approx. $5 \mathrm{~m}$ in thickness, with a very small $\mathrm{N}$ value of SPT, followed by a gravel layer with an $\mathrm{N}$-value of 50. The ground under the rail tracks was improved using chemical grouting in order to achieve greater stiffness. The excavating work was conducted in two steps.

The MEMS inclinometers were adapted for the field measurement. Figure 9 shows a photograph of the cross section of the retaining wall on the side of the downline. For MEMS inclinometers, there were five survey lines and two points at different depths were set on each line. Given the reliability of the data obtained from the field mentioned in the previous section, the MEMS inclinometers were covered by well-insulated boxes. Furthermore, nine targets for measurement using TS were set at the top of the retaining wall and measurement was conducted once a day.

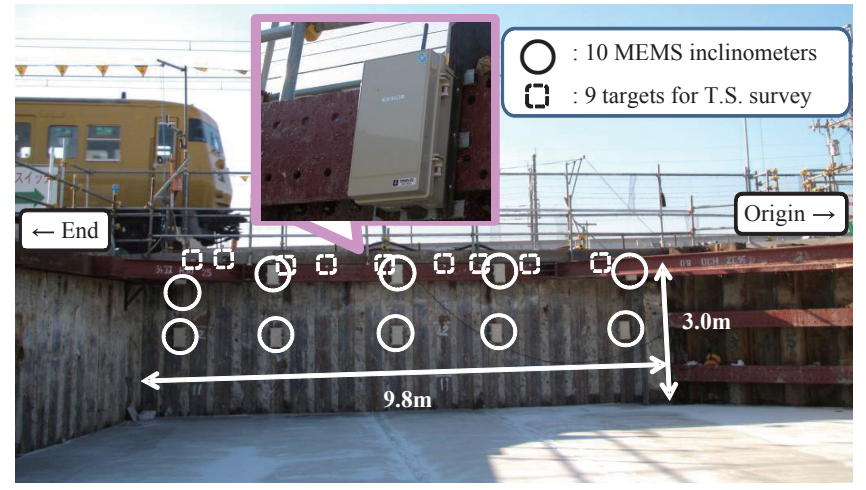

Fig. 9 Cross section along the line A-A'

\subsubsection{Evaluation of the deformation of the retaining wall}

Using the three-dimensional measurement system, the deformation of the retaining wall was evaluated and visualized based on the data obtained from the MEMS inclinometers. Figure 10 shows the deformed wall at the side of the downline after performance of excavating work.

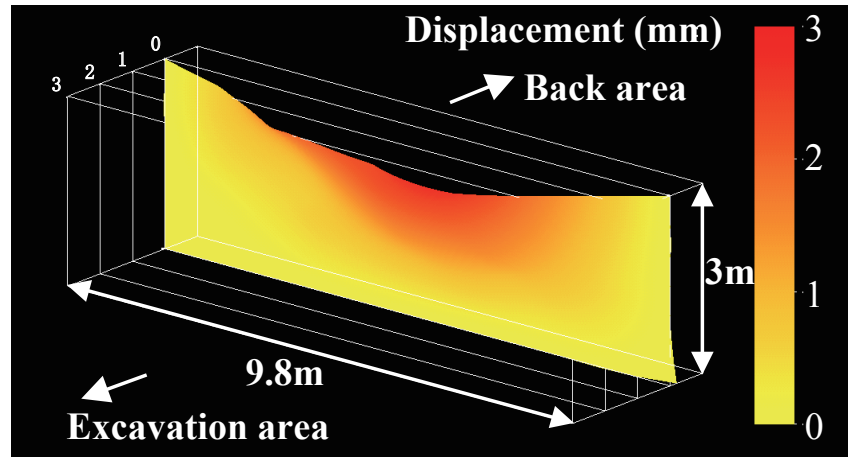

Fig. 10 Evaluated and visualized three-dimensional deformed surface of the retaining wall

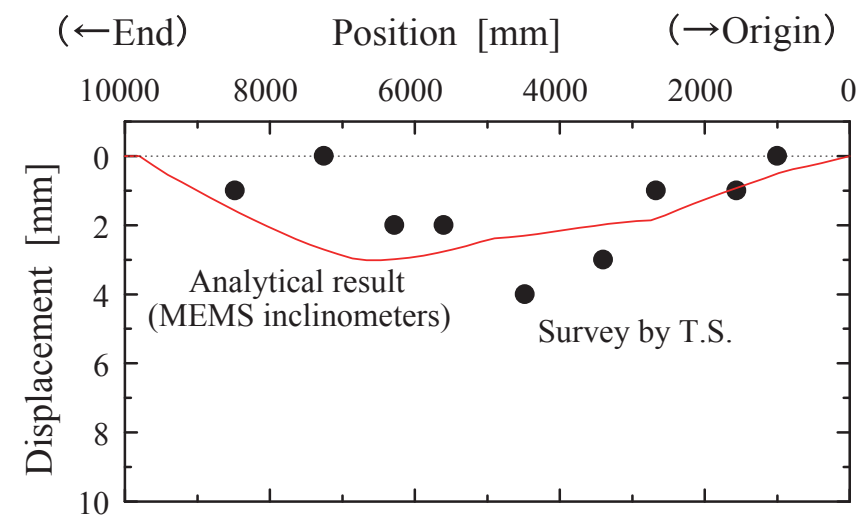

Fig. 11 Comparison of displacement at the top of the retaining wall

The simulated result could satisfactorily reproduce the deformation towards the side of the excavating area. Figure 11 provides a comparison between the simulated result, as analyzed and visualized using the three-dimensional measurement system, and the surveyed result using TS. Allowing for the measurement error of approx. several millimeters included in the survey, the simulated results coincided with the surveyed result. This simulation indicates that it was possible to evaluate the deformation of the retaining wall well using the three-dimensional measurement system in the case using stand-alone inclinometers for field measurement.

\subsubsection{Trial calculation of the cost of measurement}

For this field, the effect of the cost reduction resulting from using MEMS inclinometers was studied with a comparison with the multi-inclinometers, based on the assumption that the multi-inclinometers were set at one line and two depths. The increase in cost caused by construction close to a railroad was omitted in this trial calculation.

Figure 12 shows the cost comparison derived from the trial calculation. This figure indicates that using MEMS inclinometers for field measurement brought about a cost reduction of approx. 45\%. Because of the difference in the cost of the inclinometers and the omission of boring, measurement using MEMS inclinometers would enable costs to be reduced for measurement of retaining walls. 


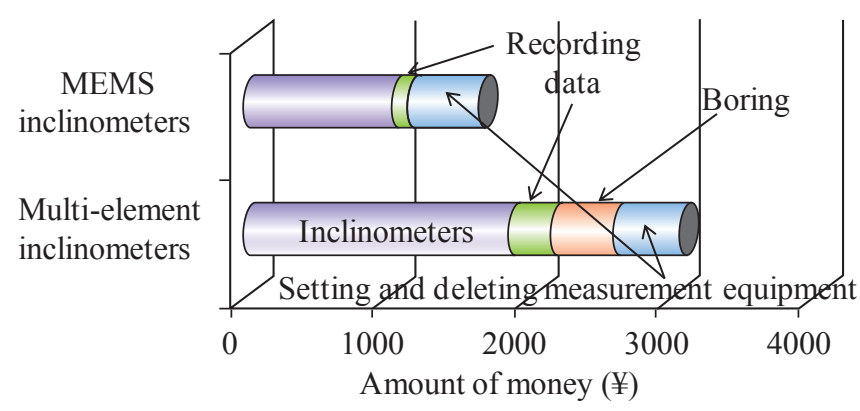

Fig. 12 Comparison of the cost of the measurement

\section{Conclusions}

In this paper, a system for evaluating and visualizing the deformation of retaining walls as a three-dimensional curved surface was developed. In order to confirm the effectiveness of the proposed system in actual monitoring, the system was applied to on-site measurement. Furthermore, a method for monitoring retaining walls by combining this system and stand-alone inclinometers was proposed. As a result, the following conclusions were reached.

(1) Based on the data obtained from measurement equipment, a method of analyzing and visualizing the deformation of retaining walls as a three-dimensional curved surface was developed. With regard to current measurement of retaining walls, the analytical method was modified to enable the data of incline to be used without conversion to displacement.

(2) The adequacy of the proposed system was examined by applying this method to measurement at a field site of excavating work. From the beginning to the end of the work, the deformation of the wall was represented satisfactorily as a three-dimensional surface. Furthermore, it was indicated that increasing the series of measured data would improve the accuracy.

(3) In order to achieve easy and economical monitoring of retaining walls using the three-dimensional measure- ment system and stand-alone inclinometers, the accuracy of the inclinations obtained from stand-alone inclinometers and their arrangement was studied. Following these studies, the proposed method was applied to field measurement with MEMS inclinometers. The deformation of the retaining wall was successfully evaluated. Furthermore, the cost of the monitoring was dramatically decreased compared with monitoring by means of measurement using multi-element inclinometers.

\section{References}

[1] Nonogaki, S., Masumoto S., and Shiono, K., "Optimal determination of geology boundary surface using cubic B-spline," Geoinformations, Vol. 19, No. 12, pp. 61-77, 2008 (in Japanese).

[2] Matsumaru, T. and Kojima, K. "Development of method for evaluating and visualizing three-dimensional deformation of earth retaining walls for excavation," Proceedings of the $18^{\text {th }}$ International Conference on Soil Mechanics and Geotechnical Engineering, 2013.

[3] Kojima, K., Matsumaru, T., Kuriyama, R., and Matsuo, N., "Observational construction using three-dimensional visualization analysis of excavating work," Proceedings of the $67^{\text {th }}$ Japanese National Conference of Civil Engineering, 2012 (in Japanese).

[4] Shiono K., Noumi Y., Masumoto S., and Sakamoto M. "Horizon2000: revised fortran program for optimal determination of geologic surfaces based on field observation including equality-inequality constraints and slope information," Geoinformations, Vol. 12, No. 4, pp. 229-249, 2001 (in Japanese).

[5] Fukuda, J., Uchimura, T., Towhata, I., Seko, I., and Wang, L. "Real-time warning for rainfall-induced slope failure by using inclinometers," Proceedings of the $42^{\text {th }}$ Japanese National Conference of Geotechnical Engineering, 2007 (in Japanese).

\section{Authors}

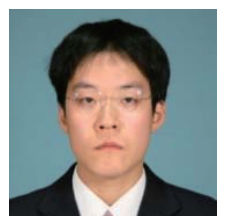

Takaki MATSUMARU

Assistant Senior Researcher, Foundation \& Geotechnical Engineering Laboratory, Structures Technology Division Research Areas: Geotechnical Engineering, Earth Structures

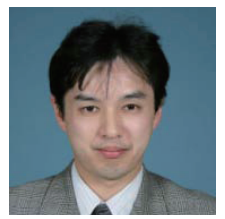

Kenichi KOJIMA, Dr. Eng. Senior Researcher, Foundation \& Geotechnical Engineering Laboratory, Structures Technology Division Research Areas: Geotechnical Engineering, Earth Structures

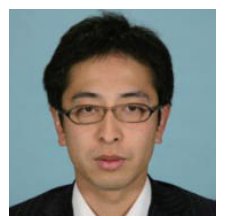

Yuji TANAKA

Senior Engineer, JR East Consultants Company, (Formerly Foundation \& Geotechnical Engineering Laboratory) Research Areas: Design of Railway Structures

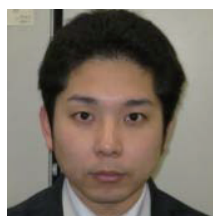

Ryosuke KURIYAMA

Chief, Fukken Engineering Co., Ltd., (Formerly Foundation \& Geotechnical Engineering Laboratory)

Research Areas: Design of Railway Structures

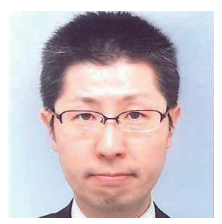

Toshiyasu HISASHIMA

Assistant Manager, East Japan Railway Company, Tokyo Construction Office Research Areas: Design of Railway Structures

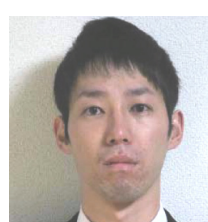

Yuki KOMINATO

Assistant Manager, West Japan Railway Company, Hiroshima Branch Research Areas: Construction Control of Railway Structures 